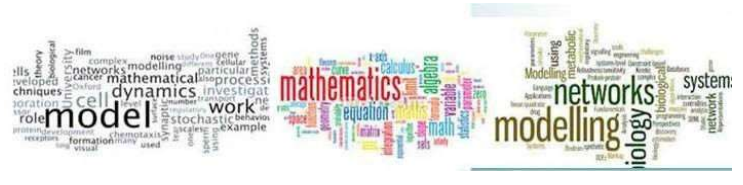

Advances in Mathematical \& computational Sciences Journal,

Vol. 7 No.1, Wach 2019

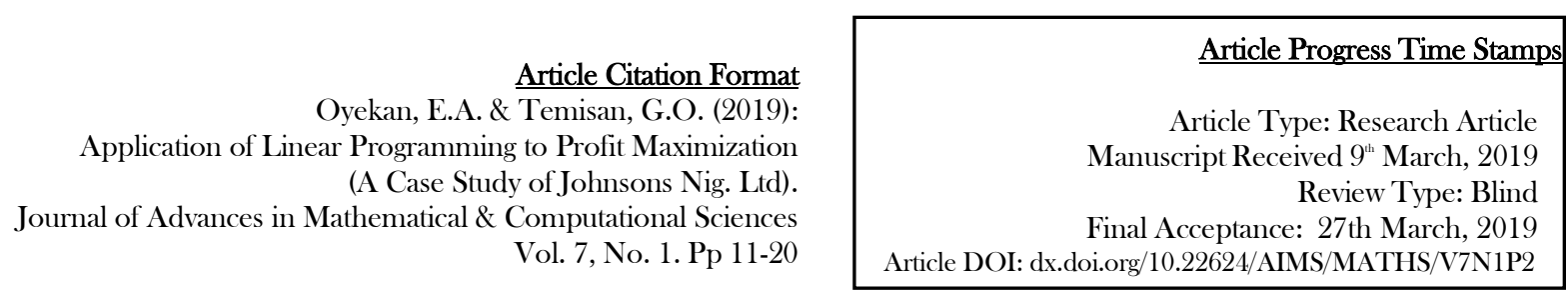

\title{
Application of Linear Programming to Profit Maximization (A Case Study of Johnsons Nig. Ltd)
}

\author{
Oyekan Ezekiel A. \& Temisan Gabriel O. \\ Department of Mathematical Sciences, \\ Ondo State University of Science and Technology (OSUSTECH), \\ Okitipupa, Ondo State, Nigeria \\ E-mail: ea.oyekan@osustech.edu.ng, temisangabriel@gmail.com
}

\begin{abstract}
Companies exist not just for the provision of goods and services but to maximize profits and drive wealth to their owners. As that is what guarantees its continuous existence, productivity and expansion. The lack of effective and sustainable profit maximization results in the liquidation of organizations, especially the small scale production companies. This work demonstrates the pragmatic use of linear programming methods in maximization of profit at Johnsons Nig. Ltd, Bakery division wherein the four various Kings Size bread were subjected to statistical analysis via the Simplex method in a bid to determine which bread size assures more objective value contribution and gives maximum profit at a given level of production capacity. The optimal solution obtained showed that the production of three units of large Kings bread size with the objective coefficient of fifty naira will yield an objective value contribution of One hundred and fifty naira. To test the feasibility of the solution, Sensitivity analysis was carried out, the result of which revealed that for the optimal solution to remain comparatively unchanged the coefficient of $x_{4}$ must remain between 50 to 30 . Otherwise, its contribution to profit will be zero, which will in turn necessitate its discontinuity.
\end{abstract}

Keyword: Linear programming, Objective functions, Constraints, Slack variables, Basic variables, Optimal solution.

\section{INTRODUCTION}

It is Impossible to properly define Linear Programming without first exploring the field of Operations research. This is because linear programming is one of the prominent techniques adopted in this field of study. Operation Research, abbreviated 'OR' for short is a scientific approach to decision making, which seeks to determine how best to design and operate a system, under conditions requiring the allocation of scarce resources (Oyekan, 2015). 


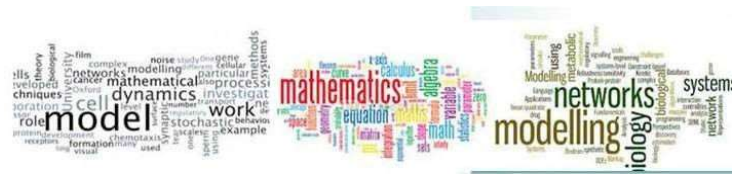

Advances in Mathematical \& computational Sciences Journal,

Vol. 7 No.1, Wach 2019

Operation research as a field of study provides a set of algorithms that acts as tools for effective problem solving and decision making in chosen application areas. OR has extensive applications in engineering, business and public systems and is also used extensively by manufacturing and service industries in decision making. The history of OR as a field of study dates back to World War II when the British military asked scientists to analyze military problems. In fact, the Second World War was perhaps the first time when people realized that scarce resources can be used effectively and allocated efficiently. [2] The application of mathematics and scientific method to military applications was called "Operations Research"; but today, it is also called "Management Science”. In general, the term Management Science also includes Operations Research; in fact, these two terms are used interchangeably.

Companies exist to maximize profits and drive wealth to their owners. As that is what guarantees its continuous existence, productivity and expansion. Businesses can only grow and expand when profit is made as this is the major reason people go into business. According to George Dantzig, one of the mathematically proven ways to ensure profit maximization is the Linear Programming Method [4]. Major management decisions involve trying to make the most effective use of Organization resources [1]. These resources include Machinery, Labour, Money, Time, Warehouse space or Raw materials to produce goods (machinery, furniture, food or cooking) or service (schedules for machinery and production, advertising, policies or investment decision). Linear Programming (LP) is a widely used mathematical technique designed to help managers in planning and decision making relative to resource allocations [3]. And also provide relatively simple and realistic solutions to these problems. A wide variety of production, finance, marketing, and distribution problems have been formulated in the linear programming framework.

Linear Programming (LP) also called Linear Optimization is a technique which is used to solve mathematical problems in which the relationships are linear in nature [9]. And as its name implies "Linear programming", it is designed for models with linear objective and constraint functions. The term "programming" indicates the solution method which can be carried out by an iterative process in which a researcher advances from one solution to a better solution, until a final solution is reached which cannot be improved upon. This final solution is termed the optimal solution of the LP problem, which is a solution that fulfills both the constraints of the problem and the set objective. A LP model can be designed and solved to determine the best course of action as in a product mix, production schedule, blending problems, etc. subject to the available constraints [7]. Generally, the objective function may be of maximization of profit (which is the focus of this research work) or minimization of costs or labor hours. Moreover, the model also consists of certain structural constraints which are set of conditions that the optimal solution should justify. Examples of the structural constraints include the raw material constraints, production time constraint, and skilled labour constraints to mention a few.

\section{LITERATURE REVIEW}

Linear Programming was developed as a discipline in 1940's, motivated initially by the need to solve complex planning problems in war time operations. This was possible by the recognition that most practical planning problems could be reformulated mathematically as finding a solution to a system of linear inequalities. The problem of solving a system of linear inequalities dates back at least as far as Fourier Joseph (1768 -1830), after whom the method of Fourier-Motzkin elimination is named. 


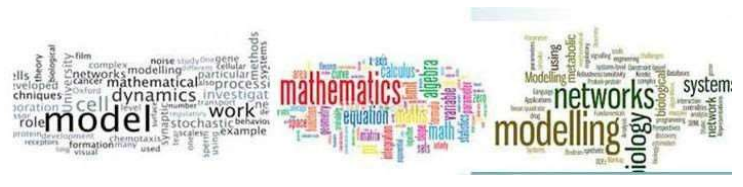

Advances in Mathematical \& computational Sciences Journal,

Vol. 7 No.1, Wach 2019

The three founders of the subject are considered to be Leonid Kantorovich, the Russian Mathematician who developed the earliest linear programming problems in 1939, George B. Dantzig, who published the simplex method in 1947, and John von Neumann, who developed the theory of the duality in the same year. The earliest linear programming was first developed by Leonid Kantorovich, a Russian mathematician, in 1939. It was used during World War II to plan expenditures and returns in order to reduce costs to the army and increase losses to the enemy. The method was kept secret until 1947 when George B. Dantzig published the simplex method and John von Neumann developed the theory of duality as a linear optimization solution, and applied it in the field of game theory. Postwar, its development accelerated rapidly as many industries found its use in their daily planning.

The Simplex method, which is used to solve linear programming, was developed by George B. Dantzig in 1947 as a product of his research work during World War II when he was working in the Pentagon with the Mil. Most linear programming problems are solved with this method. He extended his research work to solving problems of planning or scheduling dynamically overtime, particularly planning dynamically under uncertainty. This method has been the standard technique for solving a linear program since the 1940's. In brief, the simplex method passes from vertex to vertex on the boundary of the feasible polyhedron, repeatedly increasing the objective function until either an optimal solution is found, or it is established that no solution exists. In principle, the time required might be an exponential function of the number of variables, and this can happen in some contrived cases. In practice, however, the method is highly efficient, typically requiring a number of steps which is just a small multiple of the number of variables. Linear programs in thousands or even millions of variables are routinely solved using the simplex method on modern computers.

Efficient, highly sophisticated implementations are available in the form of computer software packages. In 1979, Leonid Khaciyan presented the ellipsoid method, guaranteed to solve any linear program in a number of steps which is a polynomial function of the amount of data defining the linear program. Consequently, the ellipsoid method is faster than the simplex method in contrived cases where the simplex method performs poorly. In practice, however, the simplex method is far superior to the ellipsoid method. In 1984, Narendra Karmarkar introduced an interior-point method for linear programming, combining the desirable theoretical properties of the ellipsoid method and practical advantages of the simplex method. Its success initiated an explosion in the development of interior-point methods.

These do not pass from vertex to vertex, but pass only through the interior of the feasible region. Though this property is easy to state, the analysis of interior-point methods is a subtle subject which is much less easily understood than the behavior of the simplex method. Interior-point methods are now generally considered competitive with the simplex method in most, though not all, applications, and sophisticated software packages implementing them are now available. Whether they will ultimately replace the simplex method in industrial applications is not clear.

Conclusively, the development of linear programming has been ranked among the most important scientific advances of the mid-20 ${ }^{\text {th }}$ century, and its assessment is generally accepted. Its impact since 1950 has been extra ordinary and has saved thousands or millions of dollars of many production companies.

(Wikipedia, the free encyclopedia). 


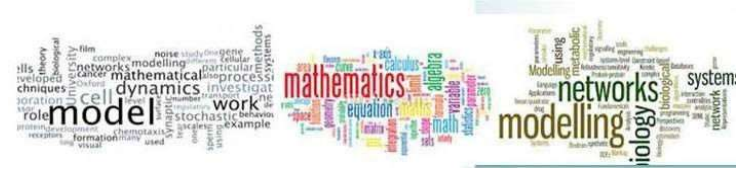

Advances in Mathematical \& computational Sciences Journal,

Vol. 7 No.1, Wach 2019

\section{METHODOLOGY}

The method adopted is the Simplex (Tableau) Algorithm. Simplex algorithm is an iterative procedure that examines the vertices of the feasible region to determine the optimal value of the objective function [6]. This method is the principal algorithm used in solving LPP consisting of two or more decision variables. It involves a sequence of exchange so that the trial solution proceeds systematically from one vertex to another in $k$, each step produces a feasible solution [5]. This procedure is stopped when the volume of $c^{\mathrm{T}} x$ is no longer increased as a result of the exchange. Listed below are the procedures required in the afore mentioned exchange:

Step 1: Setting up the Initial Simplex Tableau

In developing the initial simplex tableau, convert the constraints into equations by introducing slack variables to the inequalities. So that the problem can be re-written in standard form as a maximization problem. Such that, we can find the initial basic feasible solution by setting the decision variables $x_{1}, x_{2}, \ldots, x_{n}$ to zero in the constraints we get the basic feasible solution and the objective function becomes $Z=0$.

\section{Step 2: Optimality Process}

Having setup the initial simplex tableau, determine the entering variable (key column) and the departing variable (key row). From the $C_{j}-Z_{j}$ row we locate the column that contains the largest positive number and this becomes the Pivot Column. In each row divide the value in the R.H.S by the positive entry in the pivot column (ignoring all zero or negative entries) and the smallest one of these ratios gives the pivot row. The number at the intersection of the pivot column and the pivot row is called the pivot. Now, divide the entries of that row in the matrix by the pivot and use row operation to reduce all other entries in the pivot column, apart from the pivot, to zero.

\section{Step 3: The Stopping Criterion}

The simplex method will always terminate in a finite number of steps when the necessary condition for optimality is reached. The optimal solution to a maximum linear program problem is reached when all the entries in the net evaluation row, that is $C_{j}-Z$, are all negative or zero.

\section{DATA PRESENTATION AND ANALYSIS}

Johnsons Nigeria Limited, Bakery division, produces four sizes of Kings Bread namely: small, medium, shortlong and large. The dough for each bread size requires the following ingredients: flour, sugar, salt, yeast, butter, water, oxidizing agent and improver. The dough is baked in an electric oven at $200^{\circ} \mathrm{C}$ and lowered to $150^{\circ} \mathrm{C}$ for a fine bread crust. According to the manager, the bakery's production of each unit bread size fluctuates and presumptuously based upon the previous day market. Now, the product mix will be determined based on the data obtained in an interview with the Bakery's manager and a member of its production staff in the early month of August, 2017. 
Table 1: Bakery's (estimated) daily production capacity

\begin{tabular}{|l|l|l|l|}
\hline Bread size & $\begin{array}{l}\text { Dough input } \\
(\mathrm{kg})\end{array}$ & $\begin{array}{l}\text { Chamber capacity } \\
\left.\text { (in layers i.e. } l_{1}, l_{2}, l_{3}\right)\end{array}$ & $\begin{array}{l}\text { Production output } \\
(\text { Cups) }\end{array}$ \\
\hline Small & 150 & 200 & 600 \\
\hline Medium & 240 & 130 & 390 \\
\hline Short-Long & 340 & 110 & 330 \\
\hline Large & 600 & 100 & 300 \\
\hline
\end{tabular}

In an attempt to determine the quantity of dough required for producing a unit of each bread size, the dough input was divided by the total number of baked bread cups. The result of which is tabulated alongside the baking time (per cup), average available resources and the unit profit per cup size.

Table 2: Available resources per unit production

\begin{tabular}{|l|l|l|l|}
\hline Bread size & $\begin{array}{l}\text { Dough } \\
(\mathrm{kg})\end{array}$ & $\begin{array}{l}\text { Baking time } \\
(\text { Min) }\end{array}$ & $\begin{array}{l}\text { Profit } \\
(\mathbf{A})\end{array}$ \\
\hline Small & 0.25 & 5 & 10 \\
\hline Medium & 0.62 & 8 & 20 \\
\hline Short-Long & 1.03 & 8 & 30 \\
\hline Long & 2 & 10 & 50 \\
\hline Available resources & 175 & 30 & \\
\hline
\end{tabular}

The linear programming model can now be stated as follows:

Maximize: $Z=10 x_{1}+20 x_{2}+30 x_{3}+50 x_{4}$

Subject to: $0.25 x_{1}+0.62 x_{2}+1.03 x_{3}+2 x_{4} \leq 175$

$5 x_{1}+8 x_{2}+8 x_{3}+10 x_{4} \leq 30$

with $x_{1} \geq 0, x_{2} \geq 0, x_{3} \geq 0$ and $x_{4} \geq 0$

for all non-negative conditions

By introducing the slack variables in the objective function above the inequalities becomes equality and can thus be written as:

$$
\begin{aligned}
& \text { Maximize: } Z=10 x_{1}+20 x_{2}+30 x_{3}+50 x_{4}+0 s_{1}+0 s_{2} \\
& \text { Subject to: } 0.25 x_{1}+0.62 x_{2}+1.03 x_{3}+2 x_{4}+s_{1}=175 \\
& 5 x_{1}+8 x_{2}+8 x_{3}+10 x_{4}+s_{2}=30 \\
& x_{1} \geq 0, x_{2} \geq 0, x_{3} \geq 0 \text { and } x_{4} \geq 0 \\
& s_{1} \geq 0 \text { and } s_{2} \geq 0
\end{aligned}
$$

For which an initial simplex tableau is setup below 
Table 3: Initial Solution

\begin{tabular}{|l|l|l|l|l|l|l|l|}
\hline$B$ & $x_{1}$ & $x_{2}$ & $x_{3}$ & $x_{4}$ & $s_{1}$ & $s_{2}$ & $X_{B}$ \\
\hline$s_{1}$ & 0.25 & 0.62 & 1.03 & 2 & 1 & 0 & 175 \\
\hline$s_{2}$ & 5 & 8 & 2 & 10 & 0 & 1 & 30 \\
\hline$C_{j}-Z_{i}$ & -10 & -20 & -30 & -50 & 0 & 0 & \\
\hline
\end{tabular}

The bottom $\left(C_{j}-Z_{j}\right)$ row of table 4.3 contains the net profit per unit obtained by introducing one unit of a given variable into the solution.

Pivoting

The key column is $x_{4}$ being the least (most) negative of all entries in $\left(C_{j}-Z_{A}\right)$ row of table 3 . To obtain the key row, a ratio test is carried out as follows:

$$
\begin{gathered}
\theta_{1}=\frac{175}{2}=87.5 \\
\theta_{2}=\frac{30}{10}=3
\end{gathered}
$$

Therefore, the key row is $s_{2}$ being the least positive of the above values.

Now, to construct a new table, we find the pivotal entry at the intersection of the entry variable column and the departing variable-row. And then use the pivotal element for elimination (to get zero). Thus, we obtain a new simplex table by entering $x_{4}$ into the solution and removing $s_{2}$ variable, now (old) $R_{2}$. Performing the row operation below yields table 4

$$
\begin{gathered}
(\mathrm{New}) R_{1}: R_{1}-2 N R_{2} \\
(\mathrm{New}) R_{2}: R_{2} \times \frac{1}{10} \\
(\mathrm{New}) R_{3}: R_{3}+50 N R_{2}
\end{gathered}
$$

Table 4: First Iteration

\begin{tabular}{|l|l|l|l|l|l|l|l|}
\hline$B$ & $x_{1}$ & $x_{2}$ & $x_{3}$ & $x_{4}$ & $s_{1}$ & $s_{2}$ & $X_{B}$ \\
\hline$s_{1}$ & -0.75 & -0.98 & 0.57 & 0 & 1 & 0.2 & 169 \\
\hline$x_{4}$ & 0.5 & 0.8 & 0.8 & 1 & 0 & 0.1 & 3 \\
\hline$C_{j}-Z_{i}$ & 15 & 20 & 10 & 0 & 0 & 5 & 150 \\
\hline
\end{tabular}

Since $\left(C_{j}-Z_{\text {) }}\right.$ row of table 4 has no negative entry in the column of variables. Therefore, this is the case of optimal solution. From the $\left(X_{B}\right)$ column of the table, we have $x_{4}=3, x_{1}=x_{2}=x_{3}=0, s_{1}=169 s_{2}=0$ and the maximum value of $Z=150$.

\subsection{Results}

The linear programming model was solved using the simplex algorithm earlier discussed and the optimal solution is given as shown in Table 4. By which we have that, the production of three units of large Kings 


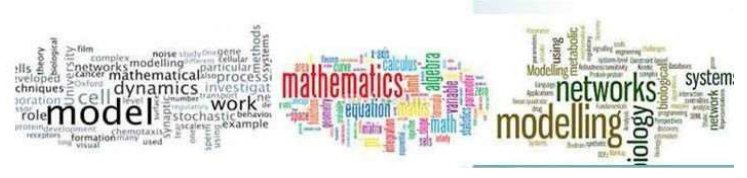

Advances in Mathematical \& computational Sciences Journal,

Vol. 7 No.1, Wach 2019

Bread size with the objective coefficient of fifty naira will give an objective value contribution of One hundred and fifty naira. This result is not substantive enough to reach a valid conclusion, thereby necessitating the need to carry out Sensitivity Analysis.

\subsection{Application of Sensitivity Analysis}

When a mathematical model is used to describe reality, approximations are made. The world as we know it is more complicated than the kinds of optimization problems that we are able to solve. Linearity assumptions usually are significant approximations. Another important approximation comes because one cannot be sure of the data being put into the model. Moreover, information may change. Sensitivity analysis is a systematic study of how sensitive solutions are to (small) changes in the data. In other words, sensitivity analysis deals with finding out the amount by which we can change the input data for the output of our linear programming model to remain comparatively unchanged [10].

The basic idea is to be able to give answers to questions of the form:

1. If the objective function coefficient changes, how does the solution change?

2. If the available resources changes, how does the solution change?

3. If a constraint is added to the problem, how does the solution change?

For the purpose of this study, we'll answer question (1) by evaluating what happens when one parameter of the problem changes.

\subsubsection{Changing the Objective function coefficient}

What we do here is to change the coefficient of the objective function and hold the constraints fixed, but only one coefficient in the objective function. We consider the linear programming model earlier formulated whose solution is, $x_{1}=x_{2}=x_{3}=0$ and $x_{4}=3$ and $Z=150$. From this solution, the relevant non-basic variables are $x_{1}, x_{2}$, and $x_{3}$.

Now, we consider what happens to the solution if the coefficient of a non-basic variable decreases. For example, if the coefficient of $x_{3}$ in the objective function was reduced from 30 to 25 . So that the objective function is:

$$
\text { Maximize: } Z=10 x_{1}+20 x_{2}+25 x_{3}+50 x_{4}
$$

the solution of which does not changes. Below, we consider the following cases:

Case 1: What happens to the solution if the coefficient of the non-basic variable is raised?

Intuitively, raising it a little bit should not matter, but raising the coefficient a lot might induce a change in the value of $x$ in a way that makes $x_{3}>0$. So, for a non-basic variable, it is expected that the solution will continue to be valid for a range (say 5 to 15) of values for coefficients of non-basic variables. If the coefficient increases enough (say $\geq 20$ ), then the solution may change. 


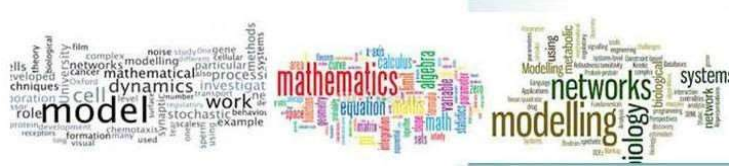

Advances in Mathematical \& computational Sciences Journal,

Vol. 7 No.1, Wach 2019

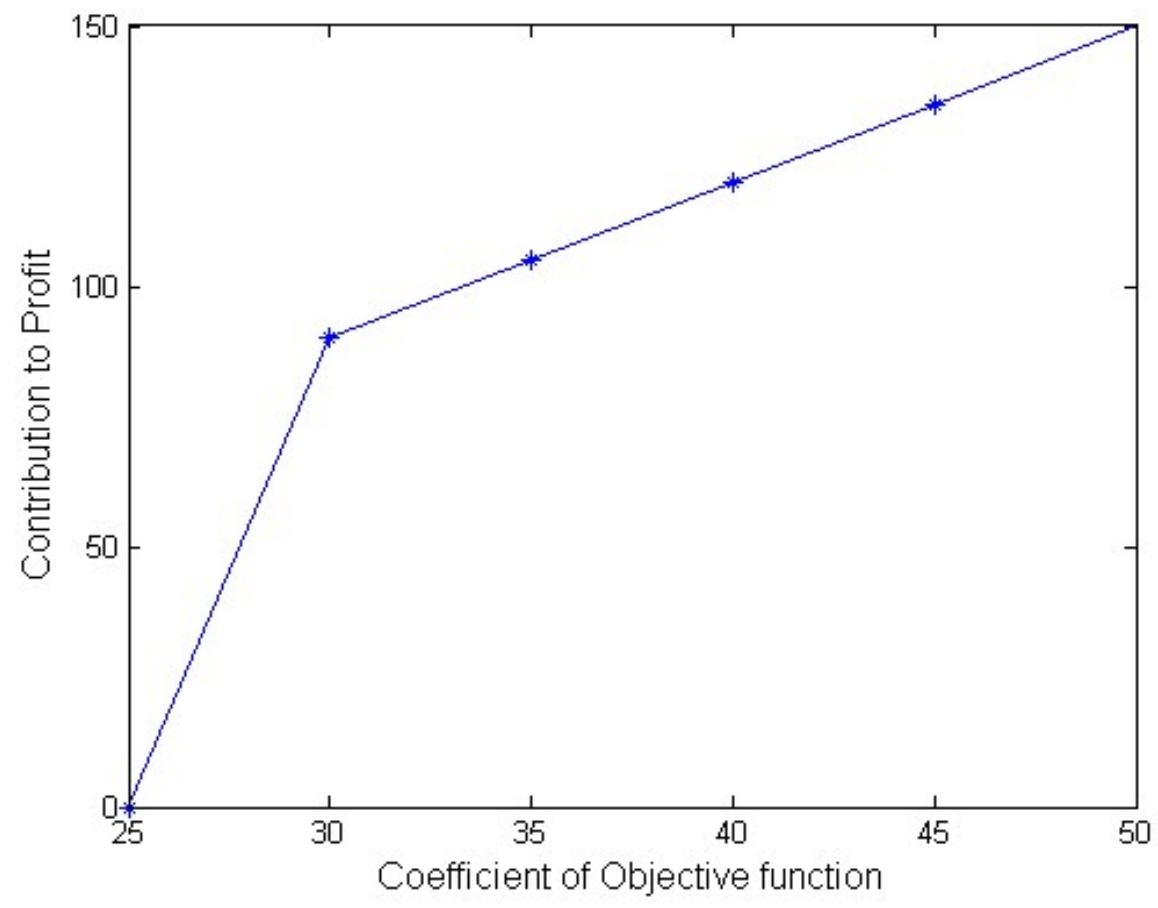

Figure 1: Changes in solution via increase in non-basic variable

Case 2: What happens to the solution if the coefficient of the basic variable decreases?

This situation differs entirely from the previous. The change makes the variable contribute less profit. It is expected that a sufficiently large reduction brings about a change in the solution. For example, if the coefficient of $x_{4}$ in the objective function of the model formulated was 30 instead of 50 . So that the objective function is:

$$
\text { Maximize: } Z=10 x_{1}+20 x_{2}+30 x_{3}+30 x_{4}
$$

for which we might (possibly) have to set $x_{4}=0$ instead of $x_{4}=3$. On the other hand, a small reduction (say 5 to 15 ) in $x_{4}{ }^{\prime} s$ objective function coefficient would typically not cause a change in the solution. In contrast to the case of the non-basic variable, such a change will have a negative impact on the value of the objective function. The value is computed by plugging in $x$ into the objective function, if $x_{4}=3$, then the coefficient of $x_{4}$ goes down from 150 to 90 (assuming that the solution remains the same).

From the above discussions, it is evident that if the coefficient of a basic variable goes up, then the value goes up and we can still use the variable. Since the value of the problem always changes whenever there is a change in the coefficient of the basic variable, intuitively, there should be a range of values of the coefficient of the objective function (a range that includes the original value) in which the solution of the problem does not change. Outside of this range, the solution will change. 


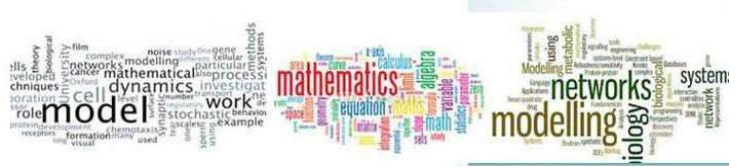

Advances in Mathematical \& computational Sciences Journal,

Vol. 7 No.1, Wach 2019

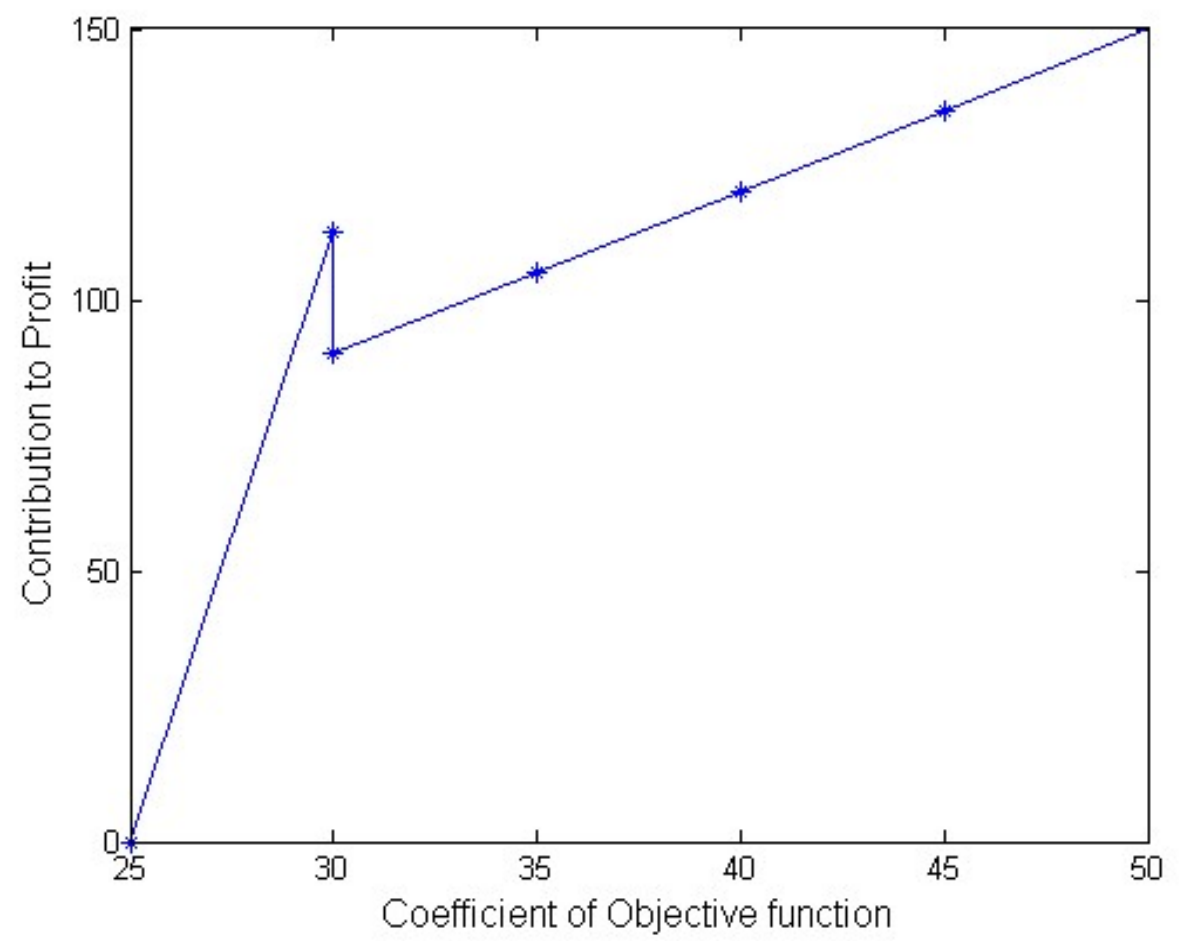

Figure 2: Changes in solution via decrease in basic variable

Based on the sensitivity analysis carried out, we now know to what extent a change in the input data induces a change in the optimal solution. And for the optimal solution to remain comparatively unchanged the coefficient of $x_{t}$ must remain between the ranges of 50 to 30 . Otherwise, its contribution to profit will be zero. Thereby changing the optimal solution, such that $x_{1}=x_{2}=x_{4}=0, x_{3}=3.75$ and $Z=112.5$.

\section{CONCLUSION}

This study has succeeded in shedding more light on the profitability of using linear programming techniques in production over any known theory of profit maximization. Based on the data obtained from the company, it was discovered that if the company is to maximize their profit, the production of large size Kings bread has to stand at three units. Since it assures an objective value contribution of one hundred and fifty naira, compared to the initial (total) contribution of one hundred and ten naira. Furthermore, the sensitivity analysis provided the range at which the optimal solution changes due to a change in the input parameter. Wherein, we considered the cases of changes in the objective function coefficient, available resources and the addition of a new constraint and how these changes affects the optimal solution. By means of this analysis, an alternative optimal solution was obtained with an objective value contribution of one hundred and twelve naira fifteen kobo, whose marginal value as compared to the original solution is thirty seven naira fifty kobo. 


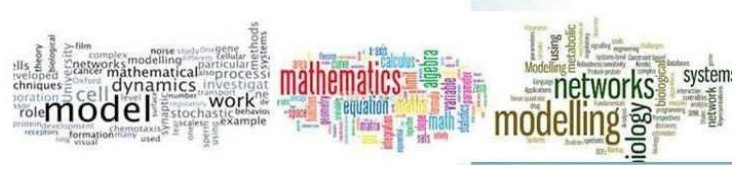

Advances in Mathematical \& computational Sciences Journal,

Vol. 7 No.1, Wach 2019

This therefore depicts that the optimal solution earlier obtained is substantive enough to reach a valid conclusion. Hence, the production of three large Kings Bread size with an objective coefficient of fifty naira assures a profit margin of one hundred and fifty naira. If only the unit production stood within the specified time range.

Thus, if there happens to be a sufficiently large reduction in the objective coefficient of the large Kings Bread size such that a unit of the short-long bread size gives a profit higher than the former, discontinuing its production is advised. Since its contribution to profit would be zero.

Researchers with interest in this subject area can consider the blending problem associated with selection of raw materials to produce the best combination at minimum cost.

\section{REFERNCES}

1. Adams W.J. (1969). Element of Linear Programming. Van Nostrand Reinhold Publishing Company International.

2. Anderson, D. Sweeny, D., Williams, T., (1995). Linear Programming Application. Quantitative method for business, $6^{\text {th }}$ edition St. Paul, minn.: West Publishing Company.

3. Bierman Jr., Bonini H., \& Charles P. (1973). Quantitative Analysis for Business Decisions. 4th edition, Richard D. Irwin, Illinois.

4. Dantzig G. B. (1963). Linear Programming and Extension. Priceton University Press.

5. Dass, H.K. (2013). Advance Engineering Mathematics 21 st Ed. New Delhi: S. Chand \& Company PVT. Ltd.

6. Hiller, F.S., Lieberman G.J. and Liebeman G. (1995). Introduction to Operations Research. New York: McGraw-Hill.

7. Nearing E.D and Tucker A.W. (1993). Linear Programming and Related Problems. Academic Press Boston.

8. Oyekan, E.A. (2015). MTH 310: Operations Research I. Lecture note, OSUSTECH (Unpublished).

9. Stroud, K.A. and Booth, D.J. (2003). Advance Engineering Mathematics $4^{\text {th }}$ edition. Houndmills, New York.

10. en.m.wikibooks.org/wiki/Operations_Research/Sensitivity_analysis 